\title{
Uso de medicina não-convencional em crianças com câncer
}

\author{
Use of unconventional medicine in children with cancer
}

\author{
Marcia C. Elias ${ }^{1}$, Elaine Alves ${ }^{2}$, Paulo Tubino ${ }^{3}$
}

\section{Resumo}

Objetivo: Um número significante de pacientes oncológicos usa ou considera usar medicina não-convencional, mas pouco se sabe sobre seu uso em crianças. Este trabalho descreve o uso de medicina não-convencional em crianças portadoras de câncer, atendidas no Hospital Universitário de Brasília/Universidade de Brasília. Método: Os pais das crianças com câncer, atendidas no Serviço de Oncologia Pediátrica do Hospital Universitário da Universidade de Brasília, entre março e agosto de 2002, foram entrevistados para a obtenção de dados referentes à prevalência e aos tipos de tratamentos não-convencionais usados, efeitos adversos, satisfação com o tratamento, e as razóes para o uso da medicina não-convencional. Resultados: Foram entrevistados 22 pais. Cerca de $55 \%$ das crianças utilizaram tratamentos não-convencionais, mais freqüentemente a fitoterapia (25\%). A indicação de terceiros foi a motivação principal para o uso da medicina não-convencional (33\%). Nenhum paciente usou medicina alternativa em substituição ao tratamento médico padrão. Quarenta e dois por cento dos pais dos usuários informaram o uso de terapias não-convencionais aos médicos dos filhos. Cinqüenta por cento dos pais disseram que eles próprios utilizavam medicina não-convencional e $95 \%$ desejavam receber mais informações a respeito dessas terapias. Conclusōes: A pesquisa confirma que os pacientes oncológicos pediátricos usam medicina não-convencional juntamente com o tratamento padrão e que a equipe médica, em geral, não é informada do emprego da medicina não-convencional pelos pacientes.

Palavras-chave: Oncologia, Pediatria, Terapias complementares.

Trabalho realizado na Universidade de Brasília.

${ }^{1}$ Enfermeira. Mestre em Ciências da Saúde pela Universidade de Brasília (UnB).

${ }^{2}$ Professora Adjunta da Área de Medicina da Criança e do Adolescente da Faculdade de Medicina (FM)/UnB. Chefe do Serviço de Oncologia Pediátrica do Centro de Cirurgia Pediátrica/Hospital Universitário de Brasília (HUB)/UnB. Doutora em Medicina pela Escola Paulista de Medicina/ UNIFESP.

${ }^{3}$ Professor Titular da Área de Medicina da Criança e do Adolescente da FM/UnB. Chefe do Centro de Cirurgia Pediátrica/HUB/UnB. Doutor e Livre-Docente pela Universidade de São Paulo.

Endereço para correspondência: SHIN, QI-2, Conj. 6, casa 4 - Cep: 71510-060 - Brasília - DF. E-mail: emo-alves@uol.com.br. 


\section{Abstract}

Background: A significant number of cancer patients use or consider using unconventional treatments, but little is known about such alternative treatments in children. This study describes the use of unconventional medicine in children with cancer at the Pediatric Oncology Service of the University Hospital/University of Brasilia. Methods: Interviews were conducted with parents of children seen in the Pediatric Oncology Service at the University of Brasilia Hospital from March to August 2002, to obtain data on the prevalence and types of unconventional therapy used, treatment satisfaction, adverse effects, and motivations for use of unconventional medicine. Results: Twenty-two parents were interviewed. Some $55 \%$ of the children had used unconventional treatment modalities, mainly herbal remedies $(25 \%)$. The principal reason for using unconventional treatment was recommendations by relatives and friends (33\%). No patient had used alternative medicine as a substitute for standard medical care. Forty-two percent of the users' parents reported the use of unconventional therapies to the children's physicians. Fifty percent of the parents themselves used unconventional medicine, and 95\% wanted more information on such therapies. Conclusions: The study confirmed that pediatric oncology patients use unconventional treatments as adjuncts to standard medical care, and that the use of these therapies is often not disclosed to the attending healthcare team.

Key words: Oncology, Pediatrics, Unconventional medicines.

\section{INTRODUÇÃO}

Desde a última década, tem sido dada uma grande atenção à medicina não-convencional. A medicina nãoconvencional compreende práticas de prevenção, diagnóstico e tratamento à margem do domínio da medicina oficial. Essas práticas não são ensinadas nas escolas médicas oficiais e, tipicamente, não são executadas nos hospitais. A medicina não-convencional pode ser dividida em duas modalidades: medicina complementar e medicina alternativa. Considera-se medicina complementar a que é usada juntamente com o tratamento padrão e que, acredita-se, contribui para obtenção dos resultados esperados com o tratamento oficial. A medicina alternativa é a utilizada em substituição ao tratamento padrão ou oficial. ${ }^{1-3}$

A prevalência do uso da medicina não-convencional tem grande variação nos diferentes países, talvez pela dificuldade em se definir exatamente medicina complementar e alternativa. ${ }^{4,5} \mathrm{Na}$ Europa, é estimada em torno de $50 \%$ e nos Estados Unidos entre 42 e $69 \% .^{1,6}$ A prevalência em nosso país ainda não está bem definida, mas o uso de terapias não-convencionais já foi relatado em cerca de $64 \%$ dos pacientes adultos com câncer, atendidos no Hospital Universitário da Universidade de Brasília.?

O paciente, diante do diagnóstico de câncer, muitas vezes, deseja experimentar o que for possível para alcançar a cura da doença. Assim, um número significativo de portadores de câncer usa ou considera a possibilidade de usar a medicina não-convencional. Existem muitas modalidades de tratamentos nãoconvencionais e, embora de uso crescente a cada dia, em geral, não há um conhecimento adequado quanto ao mecanismo de ação, eficácia e segurança dessas terapias. O paciente, por outro lado, não costuma receber qualquer esclarecimento em relação ao uso das mesmas.

Os estudos sobre medicina não-convencional, na grande maioria das vezes, focalizam o paciente adulto. Mesmo em oncologia, particularmente no Brasil, muito pouco foi abordado em relação ao uso dessa prática pelo paciente pediátrico.

O presente trabalho teve como objetivo investigar o uso de medicina não-convencional em crianças com câncer, atendidas no Serviço de Oncologia Pediátrica do Hospital Universitário da Universidade de Brasília. Foram avaliados os aspectos demográficos e socioeconômicos, assim como as razóes para o uso da medicina não-convencional, a comunicação à equipe médica sobre o uso da mesma, os tipos de terapêuticas usadas, as fontes de obtenção das referidas terapias, os possíveis efeitos adversos apresentados, se receberam orientação pela equipe médica a respeito da medicina não-convencional, e o possível desejo de receber informação a respeito dos tratamentos nãoconvencionais. Os pais das crianças arroladas no estudo foram argüidos quanto ao estado civil, escolaridade, prática religiosa, ocupação, idade e se usuários ou não de medicina não-convencional. 


\section{PACIENTES E MÉTODOS}

A população estudada constituiu-se de crianças atendidas no Serviço de Oncologia Pediátrica do Hospital Universitário da Universidade de Brasília (UnB), com idade inferior ou igual a 18 anos, apresentando doença ativa, em remissão ou recidiva, e identificadas no livro de registro de admissão de pacientes, no período de março de 2002 a agosto de 2002.

Os pais ou responsáveis deveriam tomar conhecimento e assinar o "Termo de Consentimento Livre e Esclarecido". Foram excluídos da pesquisa os pacientes portadores de neoplasias malignas em fase terminal. A entrevista com os pais foi realizada pessoalmente, após contato telefônico prévio, quando do comparecimento destes ao hospital para consulta médica ou internação de seus filhos. Em todas as entrevistas, os pais foram esclarecidos a respeito da natureza da pesquisa e do significado de medicina não-convencional.

Foi empregado o método estruturado para a coleta dos dados. Para o registro dos dados, foi elaborado, no Page Maker, versão 6.0, um plano de entrevista composto por variáveis relacionadas com os aspectos sóciodemográficos, com a história médica, e com a prática da medicina não-convencional. Os dados coletados envolveram questóes aberto-fechadas, tendo por objetivo levantar as características dos participantes da pesquisa. $\mathrm{O}$ instrumento foi preenchido exclusivamente pelo pesquisador responsável.

O presente estudo foi autorizado pelo Comitê de Ética em Pesquisa da Faculdade de Medicina da Universidade de Brasília e foram observados os preceitos éticos estabelecidos na Declaração de Helsinque e na Resolução CNS 196/96 e posteriores.

\section{RESULTADOS}

Foram entrevistados os pais de 22 crianças. Destas, $15(68,2 \%)$ eram do sexo masculino e sete $(31,8 \%)$ do sexo feminino. Do total, 11 (50\%) eram procedentes do Distrito Federal, sendo que oito $(36,4 \%)$ eram naturais de Brasília. A idade variou de 1 a 17 anos, sendo a média 7 anos e 6 meses. Em relação à escolaridade, nove $(40,9 \%)$ crianças tinham o ensino fundamental incompleto e $13(59,1 \%)$ estavam fora da idade escolar. Dezessete $(77,2 \%)$ eram pardos e cinco $(22,8 \%)$ eram brancos. A religiāo católica foi mencionada para 14 crianças $(63,7 \%)$ e a protestante para seis crianças $(27,3 \%)$. Vinte $(90,9 \%)$ crianças residiam em casas de alvenaria providas com serviço de tratamento de esgoto.

Em relação aos pais, nove $(40,9 \%)$ eram casados,
$11(50 \%)$ haviam cursado o ensino fundamental incompleto, $17(77,2 \%)$ eram pardos, $14(63,7 \%)$ eram católicos e $18(81,8 \%)$ recebiam entre um e dois salários mínimos.

Os principais aspectos sócio-demográficos estão

Tabela 1. Características sócio-demográficas das crianças atendidas no Serviço de Oncologia Pediátrica do HUB, entre março e agosto de 2002, e de seus pais

\begin{tabular}{|c|c|c|c|c|}
\hline & \multicolumn{2}{|c|}{ Crianças ( $N=22$ ) } & \multicolumn{2}{|c|}{ Pais $(N=22)$} \\
\hline & $\mathrm{N}$ & $\%$ & $\mathrm{~N}$ & $\%$ \\
\hline \multicolumn{5}{|l|}{ Sexo } \\
\hline Masculino & 15 & 68,2 & 0 & 0 \\
\hline Feminino & 7 & 31,8 & 22 & 100 \\
\hline \multicolumn{5}{|l|}{ Cor } \\
\hline Parda & 17 & 77,2 & 17 & 77,2 \\
\hline Branca & 5 & 22,8 & 5 & 22,8 \\
\hline \multicolumn{5}{|l|}{ Estado civil } \\
\hline Solteiro(a) & 22 & 100 & 5 & 22,8 \\
\hline Casado(a) & - & - & 9 & 40,9 \\
\hline Divorciado(a) & - & - & 1 & 4,5 \\
\hline Viúvo(a) & - & - & 1 & 4,5 \\
\hline Concubinato & - & - & 5 & 22,8 \\
\hline Separado(a) & & & 1 & 4,5 \\
\hline \multicolumn{5}{|l|}{ Religião } \\
\hline Católica & 14 & 63,7 & 14 & 63,7 \\
\hline Protestante & 6 & 27,3 & 7 & 31,8 \\
\hline Espírita & 1 & 4,5 & 1 & 4,5 \\
\hline Sem religião definida & 1 & 4,5 & - & - \\
\hline \multicolumn{5}{|l|}{ Escolaridade } \\
\hline Fora da idade escolar & 13 & 59,1 & - & - \\
\hline $1^{\circ}$ grau incompleto & 9 & 40,9 & 11 & 50,0 \\
\hline $1^{\circ}$ grau completo & - & - & 5 & 22,8 \\
\hline $2^{\circ}$ grau incompleto & - & - & 1 & 4,5 \\
\hline $2^{\circ}$ grau completo & - & - & 3 & 13,7 \\
\hline $3^{\circ}$ grau incompleto & - & - & 2 & 9,0 \\
\hline \multicolumn{5}{|l|}{ Renda familiar } \\
\hline Sem renda & - & - & 1 & 4,5 \\
\hline < 1 salário mínimo & - & - & 1 & 4,5 \\
\hline 1-2 salários mínimos & - & - & 18 & 82,0 \\
\hline >2 salários mínimos & - & - & 2 & 9,0 \\
\hline
\end{tabular}

resumidos na tabela 1.

Os diagnósticos estão discriminados na figura 1 . Os mais freqüentes foram o linfoma não-Hodgkin, em seis pacientes $(27,3 \%)$, e o tumor de Wilms, em cinco (22,7\%). Do total das 22 crianças do estudo, 17 (77,3\%) estavam com a doença em remissão e cinco $(22,7 \%)$ apresentavam recidiva.
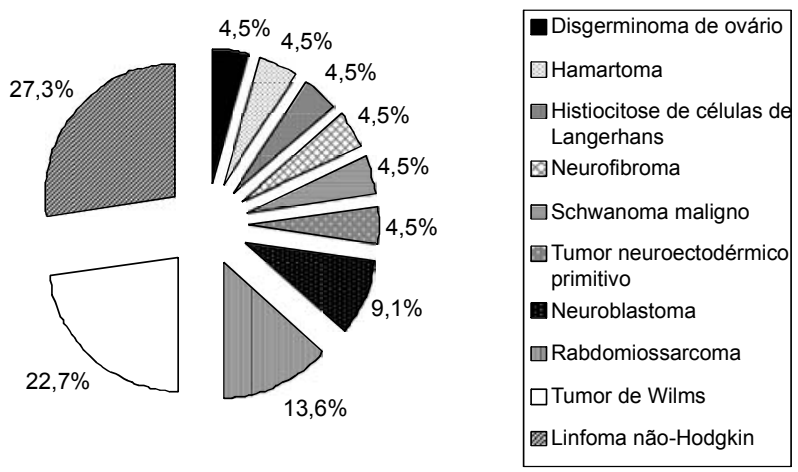

Figura 1. Diagnósticos das crianças atendidas no Serviço de Oncologia Pediátrica do HUB/UnB, entre março e agosto de 2002 
Os pais de 17 crianças $(77,2 \%)$ informaram que seus filhos ainda estavam em tratamento médico. A quimioterapia foi o tratamento mais utilizado e, isoladamente ou em combinação com outras modalidades, representou $90,9 \%$ dos tratamentos utilizados.

Nenhum dos 22 pais entrevistados recebeu orientação por parte da equipe médica a respeito da medicina não-convencional e $11(50 \%)$ informaram que eles próprios já haviam utilizado ou utilizavam tratamentos não-convencionais. Vinte e um pais $(95,5 \%)$ revelaram o desejo de esclarecimentos sobre tratamentos complementares e alternativos.

Doze crianças $(54,5 \%)$ já haviam utilizado ou utilizavam medicina não-convencional. Com relação aos usuários de medicina não-convencional, observou-se que: sete $(58,3 \%)$ eram de etnia parda; nove $(75 \%)$ seguiam a religião católica; quatro $(33,3 \%)$ eram portadores de tumor de Wilms; nove (75\%) estavam em remissão da doença e três (25\%) apresentavam recidiva. Nove (75\%) pacientes ainda estavam realizando o tratamento convencional. A fitoterapia, isoladamente (50\%) ou em combinação com outras modalidades, foi o tratamento mais usado (75\%). As modalidades de tratamentos nãoconvencionais utilizados estão especificadas na figura 2 . Uma criança $(8,3 \%)$ apresentou reação indesejável com o uso da terapia não-convencional, descrita pelo responsável como náusea.

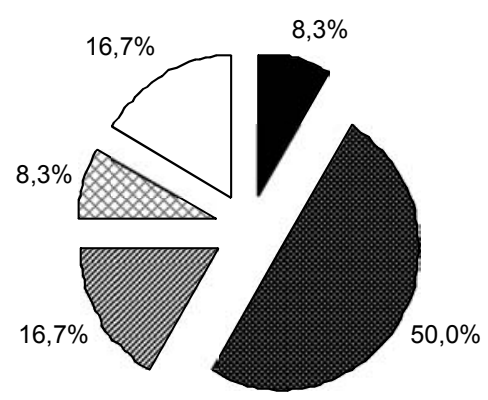

\begin{tabular}{|l|}
\hline Ervas+dieta \\
圆 Ervas \\
苗 Chás+ervas+dieta \\
凤Dieta \\
$\square$ Chás
\end{tabular}

Figura 2. Modalidades de tratamentos não-convencionais usados por crianças com neoplasia atendidas no Serviço de Oncologia Pediátrica do HUB/UnB, entre março e agosto de 2002

Dentre os pais das 12 crianças usuárias de medicina não-convencional, cinco $(41,7 \%)$ tinham o ensino fundamental incompleto, quatro $(33,3 \%)$ eram casados, sete $(58,3 \%)$ informaram que eles próprios eram usuários de medicina não-convencional e cinco $(41,7 \%)$ comunicaram ao médico que seus filhos estavam usando terapias não-convencionais. Quatro pais (33,3\%) informaram que a indicação de pessoas conhecidas, leigas foi a razão para a administração de tratamentos não-convencionais a seus filhos (figura 3) e cinco $(41,7 \%)$ relataram que obtinham as ervas usadas de terceiros (figura 4). Nenhum pai relatou ter suspendido o tratamento convencional indicado para a criança e optado por utilizar apenas a medicina não-convencional.

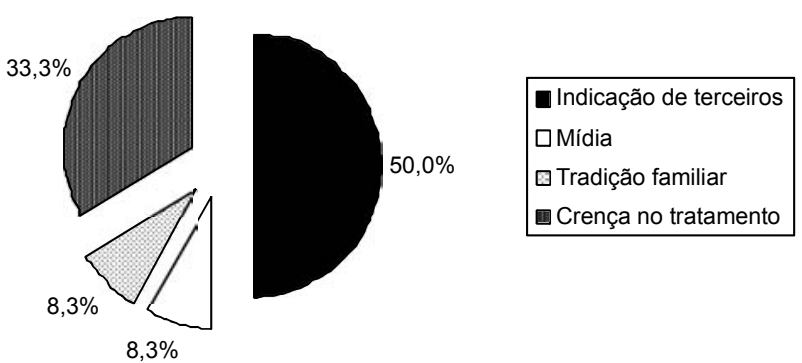

Figura 3. Razões para o uso de medicina não-convencional em crianças com neoplasia atendidas no Serviço de Oncologia Pediátrica do HUB/UnB, entre março e agosto de 2002

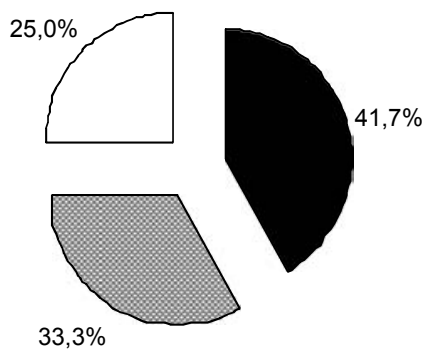

Receberam de terceiros
圆 Tinham em casa (horta)
$\square$ Compraram

Figura 4. Fontes de obtenção dos medicamentos nãoconvencionais utilizados pelas crianças com neoplasia atendidas no Serviço de Oncologia Pediátrica do HUB/UnB, entre março e agosto de 2002

\section{DISCUSSÃO}

A medicina não-convencional é utilizada para o tratamento de numerosas afecçōes, sobretudo doenças crônicas. As razões para o seu uso incluem a iatrogenia provocada pela alopatia, o custo elevado da medicina oficial, a incapacidade da medicina convencional em solucionar as doenças crônico-degenerativas e a visão de que a medicina não-convencional é mais humana e personalizada. ${ }^{6,8}$

Os defensores da medicina não-convencional acreditam que a sua popularidade se deve à visão integral do indivíduo e ao enfoque na atenção ao estado emocional e às necessidades do paciente. A maior crítica que os tratamentos não-convencionais têm sofrido diz respeito à falta de comprovação científica dos mesmos. Apesar de largamente usados, há poucas informaçōes sobre seus mecanismos de ação, eficácia e segurança. ${ }^{4,9}$ 
Nos últimos anos, tem havido um interesse maior na avaliação do uso de terapias não-convencionais pelo paciente pediátrico, principalmente no tratamento do câncer infantil. Várias pesquisas foram realizadas na Holanda, ${ }^{10}$ Austrália, ${ }^{11}$ Canadá, ${ }^{12-14}$ Dinamarca, ${ }^{15}$ GrãBretanha, ${ }^{16,17}$ Formosa $^{18} \mathrm{e}$ Estados Unidos. ${ }^{19-25} \mathrm{Na}$ literatura latino-americana, porém, os relatos são escassos. ${ }^{26}$

Esses trabalhos mostram que há um consenso quanto à constatação de que o uso de tratamentos nãoconvencionais é freqüente no paciente pediátrico, sobretudo oncológico, em percentagens que variam de 18 a 73\%, mas que, a despeito disso, os tratamentos tradicionais não são abandonados. Na maioria das vezes, o uso de terapias complementares e alternativas resultou da insatisfação com a medicina tradicional e do desejo de se fazer o que fosse possível para a cura da criança. A fitoterapia e o uso de suplementos alimentares, especialmente vitaminas em altas doses, estão entre as modalidades mais usadas em crianças. ${ }^{13,15-18,20,22,25}$ Também são citados homeopatia, ${ }^{10,12,17,21}$ tratamentos espirituais, oraçôes, técnicas de relaxamento e massagens. ${ }^{13,20,23}$ Chama atenção o fato de que mesmo para doenças pediátricas comuns são usadas terapias não-convencionais. ${ }^{18,23,25}$

O presente estudo confirmou que, em nosso meio, as crianças portadoras de câncer também são usuárias de medicina não-convencional. Entretanto, ao contrário do que se poderia supor, os pais dessas crianças não hesitaram em dar início ao tratamento oficial proposto, assim como não abandonaram o tratamento tradicional já em curso, e usaram as práticas não-convencionais de forma complementar. Além da esperança na cura do câncer, tinham a intenção, na maioria das vezes, de potencializar o resultado do tratamento convencional e amenizar seus efeitos colaterais.

Apesar de apenas uma criança no estudo ter apresentado complicações com o uso da medicina nãoconvencional, há relatos na literatura do potencial de interações indesejáveis entre os tratamentos convencional e o não-convencional. Considera-se, erroneamente, que as terapêuticas complementares e alternativas são seguras por serem naturais. Mas, ainda que naturais, não são isentas de efeitos adversos. Para algumas modalidades, como vitaminas e ervas, há a possibilidade de efeitos diretos e interações farmacodinâmicas e farmacocinéticas, quando combinadas com as terapias convencionais. ${ }^{3,27-30}$

De acordo com a literatura estrangeira, os usuários de medicina não-convencional são, em geral, pessoas de maior poder aquisitivo e maior nível cultural. ${ }^{31,32}$ No presente trabalho, as crianças faziam parte de famílias de baixo poder socioeconômico, o que está de acordo com o perfil do paciente atendido em um hospital público. Por outro lado, esta constatação indica que o interesse pelas práticas não-convencionais abrange diferentes camadas de nossa sociedade.

Como relatado em outras pesquisas, ${ }^{12,21,25}$ os pais das crianças que usavam medicina não-convencional, em sua maioria, também eram adeptos dessa mesma forma de medicina. Este pode ser um aspecto que talvez favoreça o uso de terapêuticas não-convencionais pelas crianças.

Uma proporção significativa de pais de usuários $(58,3 \%)$ não informou à equipe médica sobre o uso da medicina complementar ou alternativa, o que é igualmente relatado por, praticamente, todos os outros autores. Esta omissão pode resultar da percepção, por parte do paciente, de que os profissionais da área de saúde parecem não se importar com o fato de seus pacientes usarem tratamentos não-convencionais; do receio em abordar a prática de medicina nãoconvencional com a equipe médica por conta da reação contrária esperada; do conceito de que os profissionais de saúde carecem de conhecimento a respeito das terapêuticas não-convencionais e, também, porque a oferta de práticas de medicina não-convencional se multiplica livremente a cada dia, mesmo que não seja acompanhada de uma verdadeira informação para a saúde. Neste último caso, é necessário considerar o papel das autoridades na normatização, segurança e fiscalização da prática de medicina não-convencional.

Mais de $95 \%$ dos pais entrevistados manifestaram o desejo de receber esclarecimentos a respeito das terapias não-convencionais, o que já havia sido detectado por outros autores. ${ }^{12,14,16}$ Acreditamos que o profissional de saúde deva abordar o uso da medicina não-convencional com uma atitude neutra e com informação bem dirigida. É recomendável o registro do uso de tratamentos nãoconvencionais no prontuário do paciente.

$\mathrm{O}$ grande interesse e o uso significativo de medicina não-convencional são um desafio para os profissionais de saúde, que necessitam estar atentos quanto às possíveis interações indesejáveis e aos efeitos colaterais desses tratamentos. Somente com um conhecimento adequado do mecanismo de ação, eficácia e segurança das terapias complementares e alternativas é que poderão informar e esclarecer seus pacientes. ${ }^{33,34}$ Idealmente, esses tratamentos deveriam ser submetidos a processos rigorosos de investigação, já que não é desejável incluir as terapêuticas não-convencionais no plano de cuidados do paciente sem uma análise cuidadosa.

Achamos importante enfatizar que a equipe médica deve estar alertada para o fato de que seus pacientes e os pais de seus pacientes, em geral, não comunicam 
que estão usando medicina não-convencional em adição ao tratamento prescrito. Tanto a equipe médica quanto os pais devem estar informados a respeito dos benefícios e dos efeitos adversos dessas práticas para que possam oferecer o melhor tratamento à criança, preservando a continuidade do tratamento convencional já consagrado.

\section{REFERÊNCIAS BIBLIOGRÁFICAS}

1. Richardson MA, Sanders T, Palmer JL, Greisinger A, Singletary SE. Complementary/alternative medicine use in a comprehensive cancer center and the implications for oncology. J Clin Oncol. 2000;18:2505-14.

2. Ernst E. The role of complementary and alternative medicine. BMJ. 2000;321:1133-5.

3. Ernst E. Evaluating complementary and alternative therapies for cancer patients. CA Cancer J Clin. 1999;49:362-75.

4. Cassileth BR. Evaluating complementary and alternative therapies for cancer patients. CA Cancer J Clin. 1999;49:362-75.

5. Penson RT, Vastro CM, Seiden MV, Chabner BA, Lynch TJ Jr. Complementary, alternative, integrative, or unconventional medicine? Oncologist. 2001;6:463-73.

6. Astin JA. Why patients use alternative medicine: results of a national study. JAMA. 1998 May 20;279(19):1548-53.

7. Elias MC, Alves E. Medicina não-convencional: prevalência em pacientes oncológicos. Rev Bras Cancerol. 2002;48:523-32.

8. Beyerstein BL. Alternative medicine and common errors of reasoning. Acad Med. 2001;76:230-7.

9. Nahin RL, Straus SE. Research into complementary and alternative medicine: problems and potential. BMJ. 2001;322:161-4.

10. Grootenhuis MA, Last BF, de Graaf-Nijkerk JH, van der Wel M. Use of alternative treatment in pediatric oncology. Cancer Nurs. 1998;21:282-8.

11. Sawyer MG, Gannoni AF, Toogood IR, Antoniou G, Rice $M$. The use of alternative therapies by children with cancer. Med J Aust. 1994 Mar 21;160:320-2.

12. Spigelblatt L, Laine-Ammara G, Pless IB, Guyver A. The use of alternative medicine by children. Pediatrics. 1994;94(6 Pt 1):811-4.

13. Fernandez CV, Stutzer CA, MacWilliam L, Fryer C. Alternative and complementary therapy use in pediatric oncology patients in British Columbia: prevalence and reasons for use and nonuse. J Clin Oncol. 1998;16:1279-86.

14. Bold J, Leis A. Unconventional therapy use among children with cancer in Saskatchewan. J Pediatr Oncol Nurs. 2001;18:16-25.

15. Madsen H, Andersen S, Nielsen RG, Dolmer BS, Host A,
Damkier A. Use of complementary/alternative medicine among paediatric patients. Eur J Pediatr. 2003 May;162(5):334-41. Epub 2003 Feb 27.

16. Molassiotis A, Cubbin D. 'Thinking outside the box': complementary and alternative therapies use in paediatric oncology patients. Eur J Oncol Nurs. 2004 Mar;8(1):50-60.

17. Simpson N, Roman K. Complementary medicine use in children: extent and reasons. A population-based study. $\mathrm{Br}$ J Gen Pract. 2001 Nov;51(472):914-6.

18. Yeh CH, Tsai JL, Li W, Chen HM, Lee SC, Lin CF, et al. Use of alternative therapy among pediatric oncology patients in Taiwan. Pediatr Hematol Oncol. 2000 JanFeb;17(1):55-65.

19. Kelly KM, Jacobson JS, Kennedy DD, Braudt SM, Mallick M, Weiner MA. Use of unconventional therapies by children with cancer at an urban medical center. J Pediatr Hematol Oncol. 2000;22:412-6.

20. McCurdy EA, Spangler JG, Wofford MM, Chauvenet AR, McLean TW. Religiosity is associated with the use of complementary medical therapies by pediatric oncology patients. J Pediatr Hematol Oncol. 2003 Feb;25:125-9.

21. Pitetti R, Singh S, Hornyak D, Garcia SE, Herr S. Complementary and alternative medicine use in children. Pediatr Emerg Care. 2001 Jun;17(3):165-9.

22. Neuhouser ML, Patterson RE, Schwartz SM, Hedderson MM, Bowen DJ, Standish LJ. Use of alternative medicine by children with cancer in Washington state. Prev Med. 2001 Nov;33(5):347-54.

23. Friedman T, Slayton WB, Allen LS, Pollock BH, DumontDriscoll M, Mehta P, et al. Use of alternative therapies for children with cancer. Pediatrics. 1997;100(6):E1.

24. Davis MP, Darden PM. Use of complementary and alternative medicine by children in the United States. Arch Pediatr Adolesc Med. 2003;157:393-6.

25. Ottolini MC, Hamburger EK, Loprieato JO, Coleman RH, Sachs HC, Madden R, Brasseux C. Complementary and alternative medicine use among children in the Washington, DC area. Ambul Pediatr. 2001;1(2):122-5.

26. Hapner LRM, Kato MADP, Cassilha A, Abrão D, Tanaka E, Mortean CR, et al. Medicina alternativa em oncologia pediátrica. In: Liga Paranaense de Combate ao Câncer. Informe científico. Curitiba, Centro de Projetos de Ensino e Pesquisa, 1987. p.113-21.

27. Izzo AA, Ernst E. Interactions between herbal medicines and prescribed drugs: a systematic review. Drugs. 2001;61:2163-75.

28. Stedman C. Herbal hepatotoxicity. Semin Liver Dis. 2002;22:195-206. 
29. Niggemann B, Gruber C. Side-effects of complementary and alternative medicine. Allergy. 2003;58:707-16.

30. Jankovic M, Spinetta JJ, Martins AG, Pession A, Sullivan M, D'Angio GJ, et al. Non-conventional therapies in childhood cancer: guidelines for distinguishing nonharmful from harmful therapies: a report of the SIOP
Working Committee on Psychosocial Issues in Pediatric Oncology. Pediatr Blood Cancer. 2004 Jan;42(1):106-8.

31. Cassileth BR Complementary therapies: the American experience. Support Care Cancer. 2000;8:16-23.

32. Cassileth BR, Deng G. Complementary and alternative therapies for cancer. Oncologist. 2004;9(1):80-9. 